\title{
Auditory and Dynamic Modeling Paradigms to Detect L2 Mispronunciations
}

\author{
Christos Koniaris, Olov Engwall and Giampiero Salvi \\ Centre for Speech Technology, School of Computer Science \& Communication \\ KTH - Royal Institute of Technology, Stockholm, Sweden \\ [koniaris, engwall, giampi] akth.se
}

\begin{abstract}
This paper expands our previous work on automatic pronunciation error detection that exploits knowledge from psychoacoustic auditory models. The new system has two additional important features, i.e., auditory and acoustic processing of the temporal cues of the speech signal, and classification feedback from a trained linear dynamic model. We also perform a pronunciation analysis by considering the task as a classification problem. Finally, we evaluate the proposed methods conducting a listening test on the same speech material and compare the judgment of the listeners and the methods. The automatic analysis based on spectro-temporal cues is shown to have the best agreement with the human evaluation, particularly with that of language teachers, and with previous plenary linguistic studies. Index Terms: L2 pronunciation error, auditory model, linear dynamic model, distortion measure, phoneme.
\end{abstract}

\section{Introduction}

People who learn a foreign language often experience the difficulty of this process. Concentrating on the pronunciation complexity (avoiding other factors, such as grammatical rules, unknown vocabulary etc.), we develop automatic, language independent, diagnostic evaluation methods to identify the problematic phonemes for individual speakers from different language backgrounds. In our previous work [1], we presented a novel auditory model-based approach to account for second language (L2) pronunciation assessment. The method calculates the dissimilarity of the distortion's Euclidean metric in the high-dimensional native auditory space and non-native acoustic signal space and compares it to the native only setting in order to find phonemes for which there is an additional dissimilarity for the foreign speaker. The method thus relies on perceptual exploitation of the native speech signal rather than a classifier to detect mispronunciations.

In this work, we expand the above scheme in two directions: i) by using a spectro-temporal auditory model and ii) by including classification feedback from a linear dynamic model (LDM). Both alterations satisfy our objective to include dynamic information from the speech signal which enriches the system with useful knowledge. Moreover, we study the LDM independently, and by using two different observation sequences, we analyze the detected pronunciation errors. Finally, we perform a listening test on the same data with native listeners to verify the accuracy of our methods.

\section{Hybrid pronunciation diagnosis method}

In this section, we describe the new method for automatic pronunciation error detection that combines information from an auditory model and an LDM.

\subsection{Modeling native perception}

Fig. 1 shows the proposed scheme for modeling perception of a native speech signal on the phone level. The fundamental idea is to combine perceptual information from models of the human auditory periphery with knowledge acquired from classification. A phone-level transcription is first automatically generated from the native speech signal and the accompanying text file using an HMM-based aligner [2]. These phonelevel transcription files are used to separate the speech data into phoneme categories which then serve as input $\mathbf{s}_{n}$ to the auditory model, where $\mathbf{s}_{n}$ is the time domain native speech signal. The auditory model output $\mathbf{y}_{n}$, also known as the internal representations, are computed on a frame basis. A finite set of perturbations $\hat{\mathbf{s}}_{n}$ are created in a small region around each input vector. Finally, a Euclidean norm-based distance measure $v_{n}\left(\mathbf{s}_{n_{I}}, \hat{\mathbf{s}}_{n_{I, j}}\right)=\left\|\mathbf{y}\left(\mathbf{s}_{n_{I}}\right)-\mathbf{y}\left(\hat{\mathbf{s}}_{n_{I, j}}\right)\right\|^{2}$ is used to calculate the perceptually-relevant distortion indicated by the auditory model, where $I \in \mathcal{I}$ and $j \in \mathcal{J}_{i}$ represent a finite frame sequence and a finite set of acoustic perturbations, respectively.

In parallel, the speech waveform signal is transformed into mel frequency cepstrum coefficients (MFCCs) $\mathbf{c}_{n}$ which are then considered to be the observation vector of an LDM described by the following pair of equations

$$
\begin{gathered}
\mathbf{x}_{n_{k+1}}=\mathbf{F} \mathbf{x}_{n_{k}}+\mathbf{w}_{k} \\
\mathbf{c}_{n_{k}}=\mathbf{H} \mathbf{x}_{n_{k}}+\mathbf{u}_{k},
\end{gathered}
$$

where $\mathbf{x}_{n_{k}}, \mathbf{c}_{n_{k}}$ are the state and the observation vectors at the time frame $k$, respectively, and $\mathbf{w}_{k}, \mathbf{u}_{k}$ are uncorrelated, zeromean Gaussian vectors with covariances $E\left\{\mathbf{w}_{k} \mathbf{w}_{l}^{T}\right\}=\mathbf{P} \delta_{k l}$ and $E\left\{\mathbf{u}_{k} \mathbf{u}_{l}^{T}\right\}=\mathbf{R} \delta_{k l}$. The initial state $\mathbf{x}_{n_{0}}$ is Gaussian with known mean $\mu_{\mathbf{x}_{n_{0}}}$ and covariance $\Sigma_{\mathbf{x}_{n_{0}}}$. Eq. (1) describes the state dynamics, and Eq. (2) gives an observation prediction based on the state estimation. The parameters of the $\operatorname{LDM} \theta=\{\mathbf{F}, \mathbf{H}, \mathbf{P}, \mathbf{R}\}$ are calculated using an ExpectationMaximization (EM) based algorithm introduced in [3]. Given the observation vectors $\mathbf{C}_{n}=\left[\mathbf{c}_{n_{0}} \ldots \mathbf{c}_{n_{\mathcal{I}}}\right]$ and the state vectors $\mathbf{X}_{n}=\left[\mathbf{x}_{n_{0}} \ldots \mathbf{x}_{n_{\mathcal{I}}}\right]$, the maximum likelihood (ML) estimates of $\theta$ are obtained by minimizing

$$
\begin{array}{r}
L\left(\mathbf{X}_{n}, \mathbf{C}_{n}, \theta\right)= \\
-\sum_{k=1}^{\mathcal{I}}\left\{\log |\mathbf{P}|+\left[\mathbf{x}_{n_{k}}-\mathbf{F} \mathbf{x}_{n_{k-1}}\right]^{T} \mathbf{P}^{-1}\left[\mathbf{x}_{n_{k}}-\mathbf{F} \mathbf{x}_{n_{k-1}}\right]\right\} \\
-\sum_{k=0}^{\mathcal{I}}\left\{\log |\mathbf{R}|+\left[\mathbf{c}_{n_{k}}-\mathbf{H} \mathbf{x}_{n_{k}}\right]^{T} \mathbf{R}^{-1}\left[\mathbf{c}_{n_{k}}-\mathbf{H} \mathbf{x}_{n_{k}}\right]\right\} .
\end{array}
$$

Using Eq. (1), the state dynamics representations $\mathbf{x}_{n}$ are computed and the native state representation distortion measure $\varphi_{n}\left(\mathbf{s}_{n_{I}}, \hat{\mathbf{s}}_{n_{I, j}}\right)=\left\|\mathbf{x}\left(\mathbf{s}_{n_{I}}\right)-\mathbf{x}\left(\hat{\mathbf{s}}_{n_{I, j}}\right)\right\|^{2}$ is calculated. Finally, 


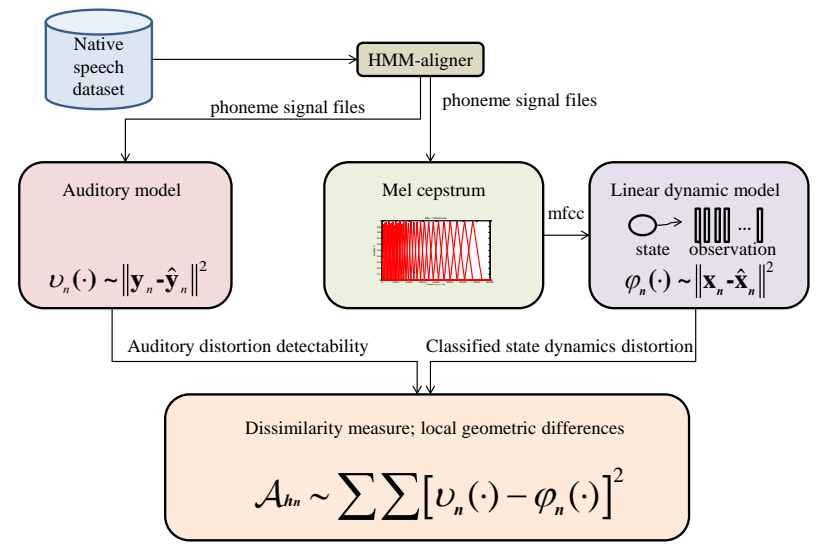

Figure 1: Block diagram of the hybrid method using native speech signal. The $L^{2}$ norm of the phone distortion is evaluated in an auditory model domain. In parallel, an LDM is trained using native speech to perform a phone classification, and the corresponding $L^{2}$ norm is then evaluated in the LDM state domain. The objective is to measure the Euclidean geometry dissimilarity in the considered domains.

the dissimilarity measure for the native speech signal is evaluated as

$$
\mathcal{A}_{h_{n}}=\frac{1}{\mathcal{I}} \sum_{I \in \mathcal{I}} \frac{1}{\mathcal{J}_{i}} \sum_{j \in \mathcal{J}_{i}}\left[v_{n}\left(\mathbf{s}_{n_{I}}, \hat{\mathbf{s}}_{n_{I, j}}\right)-\varphi_{n}\left(\mathbf{s}_{n_{I}}, \hat{\mathbf{s}}_{n_{I, j}}\right)\right]^{2} .
$$

We note that the above $\mathcal{A}_{h_{n}}$ differs from the corresponding measure $\mathcal{A}_{n}$ in [1] because it additionally combines classification knowledge from the statistical dynamic model for the native speech signal.

\subsection{Perceptual assessment of non-native speech}

We next modify the above scheme to deal with non-native speech signals. The procedure described in Sec. 2.1 for alignment, perturbation of the signal $\mathbf{s}_{\ell}$ to create nearby sounds $\hat{\mathbf{s}}_{\ell}$ and generation of MFCCs are applied to non-native speech data.

The non-native acoustic representation distortion measure $\varphi_{\ell}\left(\mathbf{s}_{\ell_{I}}, \hat{\mathbf{s}}_{\ell_{I, j}}\right)=\left\|\mathbf{c}\left(\mathbf{s}_{\ell_{I}}\right)-\mathbf{c}\left(\hat{\mathbf{s}}_{\ell_{I, j}}\right)\right\|^{2}$ is calculated and compared with the native perceptual distortion in the dissimilarity measure

$$
\mathcal{A}_{\ell}=\frac{1}{\mathcal{I}} \sum_{I \in \mathcal{I}} \frac{1}{\mathcal{J}_{i}} \sum_{j \in \mathcal{J}_{i}}\left[v_{n}\left(\mathbf{s}_{n_{I}}, \hat{\mathbf{s}}_{n_{I, j}}\right)-\varphi_{\ell}\left(\mathbf{s}_{\ell_{I}}, \hat{\mathbf{s}}_{\ell_{I, j}}\right)\right]^{2} .
$$

In [1], we presented a novel technique so as to be able to compute Eq. (5) - a direct calculation of which would otherwise request both $\mathbf{s}_{n}$ and $\mathbf{s}_{\ell}$ to have the same number of frames. The underlying idea is to compute $\varphi_{\ell}\left(\mathbf{s}_{\ell_{I}}, \hat{\mathbf{s}}_{\ell_{I, j}}\right)$ in a non-native subspace inside the native speech signal representation domain, noting that a speech signal representation is a mapping from a phone $\mathbf{p}$ to the acoustic feature domain, $\mathbf{c}_{n}$ for the native speech or $\mathbf{c}_{\ell}$ for the non-native speech signals. We use a statistical approach, described in [1], to estimate the relation between the native and non-native speech signal representations by considering the distortion of all frames of a specific language group to follow a Gaussian distribution.

In the final stage, the examined target phoneme is evaluated according to what we call, hybrid native perceptual assessment degree $(h$-nPAD) as

$$
\Xi_{h_{\ell}}=\frac{\mathcal{A}_{\ell}}{\mathcal{A}_{h_{n}}}
$$

considering a mispronunciation to have occurred when $\Xi_{h_{\ell}}>1$

\section{LDM pronunciation analysis method}

In this second approach, the problem of L2 mispronunciation is treated as a classification problem and therefore we use directly the LDM system to find the mispronounced phonemes. We aim not only to detect errors, but also to find the phonemes that substitute the targets. We use two speech signal representations, namely the modified MFCCs (MMFCCs) [4] and the classical MFCCs [5]. The MMFCCs are auditory motivated MFCCs optimized in an offline manner based on the assumption that the local geometries of the feature domain and the perceptual auditory domain [6] should be similar. These features have been used in speech recognition systems resulting in a significant improvement under clean and noisy conditions, in comparison to the conventional MFCCs [4]. We hence use these features so as to investigate if the LDM system benefits from a more modern perceptually-relevant observation input vector.

The native speech features (MMFCCs or MFCCs) $\mathbf{c}_{n}$ are used to train the phoneme models. We set the size of the statespace (state vectors) to be equal to the size of the observation vectors. The initial state-transition matrices, and the covariance of the initial state $\mathbf{x}_{n_{0}}$ are directly estimated from the observation vectors. The noise covariance matrices of the system are randomly initialized. The EM algorithm is performed in 5 iterations. During the expectation step, we compute the forward - backward counts from the Kalman smoother and collect the sufficient statistics $[7,8]$. In the maximization step we obtain the new estimates for the system parameters. Additionally, we compute the cross covariances in both the forward and the backward pass [3]. Since the estimates of the parameters of the state linear equation are mutually dependent, we consider an additional iterative estimation process based on the same sufficient statistics obtained in the expectation step. This iterative process is terminated when a predefined (small) threshold of the distance $d(\theta, \hat{\theta})=\frac{\|\theta-\hat{\theta}\|}{\|\theta\|}$ between two successive estimates $\theta$ and $\hat{\theta}$ is reached.

The classification process begins by considering the nonnative signal representations $\mathbf{c}_{\ell}$ of a specific phoneme class and the parameters of each phoneme category $\theta$ that were previously estimated during the training stage using the native speech signal. The data are classified according to the value of the loglikelihood

$$
L\left(\mathbf{C}_{\ell}, \theta\right)=-\sum_{k=0}^{\mathcal{I}}\left\{\log \left|\Sigma_{e_{k}}(\theta)\right|+e_{k}^{T}(\theta) \Sigma_{e_{k}}^{-1}(\theta) e_{k}(\theta)\right\},
$$

where $e_{k}(\theta)$ is the prediction error and $\Sigma_{e_{k}}(\theta)$ its covariance, obtained from the linear quadratic estimation.

\section{Experimental setup}

To include the spectro-temporal auditory processing of the native speech we used the so called Dau model [9] to compute the distortion in the native auditory domain. In parallel, we considered the static and dynamic MFCCs to calculate the distortion in the first language (L1) or L2 acoustic domains for the native or non-native speech, respectively. To do so, we considered a relatively large frame $I$ of $40 \mathrm{~ms}$ with an overlap of $10 \mathrm{~ms}$ to compute the auditory model output $\mathbf{y}_{n}\left(\mathbf{s}_{n_{I}}\right)$ for the native speech signal $\mathbf{s}_{n_{I}}$. Each such frame $I$ consisted of three overlapping subframes $i-1, i$ and $i+1$ of $20 \mathrm{~ms}$ each with an overlap of 10 
Table 1: Problematic phonemes per language background. The phonemes are shown in decreasing order, starting from the one with the highest nPAD value. Phonemes that differ from the linguistic study [10] are listed in parentheses. Phonemes that were included in our listening test are underlined, and additionally shown in bold if the experts' opinion agree with the automatic evaluation.

\begin{tabular}{|c|c|c|c|}
\hline L1 bkgr. & evaluat. & vowels & consonants \\
\hline \multirow[t]{2}{*}{ English (US) } & $\Xi_{\ell}$ & 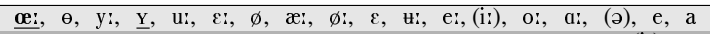 & $(\underline{\mathbf{b}}),(\mathrm{p}),(\mathrm{d}), \mathrm{f}, \mathrm{t}, \mathrm{j}, \mathrm{s},(\mathrm{g}), \mathrm{k}, \mathrm{t},(\mathrm{h}),(\mathrm{v})$ \\
\hline & $\Xi_{h_{\ell}}$ & 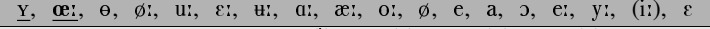 & (b), (p), t, (d), k, (g), (j), t, (h), $6, s, s$ \\
\hline \multirow[t]{2}{*}{ German } & $\Xi_{\ell}$ & 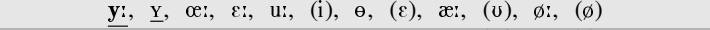 & $\underline{\mathbf{b}}, \mathrm{t}, \mathrm{d}, \mathrm{j}, \mathrm{s}, \mathrm{v},(\mathrm{h}), \mathrm{g}, \mathrm{n}, \mathrm{f}, \mathrm{k}, \mathrm{t}, \mathrm{s}, \mathrm{r}, \mathrm{p},(\mathrm{m}), \mathrm{j}$ \\
\hline & $\Xi_{h_{\ell}}$ & 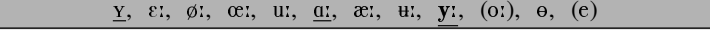 & $\underline{\mathbf{b}}, \mathrm{d}, \mathrm{t}, \mathrm{p}, \mathrm{g}, \mathrm{j}, \mathrm{k}, \mathrm{t},(\mathrm{h}), \mathrm{s}, \mathrm{s}, \mathrm{c}, \mathrm{r},(\mathrm{l}),(\mathrm{m}), \mathrm{v}, \mathrm{J}$ \\
\hline \multirow[t]{2}{*}{ French } & $\Xi_{\ell}$ & 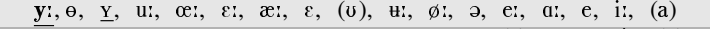 & $\underline{\mathbf{d}}, \mathrm{b}, \mathrm{t}, \mathrm{f}, \mathrm{s}, \mathrm{g}, \mathrm{j}, \mathrm{p},(\mathrm{v}), \mathrm{k}, \mathrm{t}, \mathrm{r}, \mathrm{h}, \mathrm{n}, \mathrm{s}$, (l) \\
\hline & $\Xi_{h_{\ell}}$ & 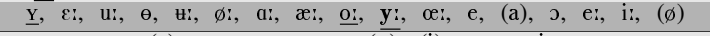 & $\underline{\mathbf{d}}, \mathrm{b}, \mathrm{t}, \mathrm{p}, \mathrm{g}, \mathrm{k},(\mathrm{j}), \mathrm{t}, \mathrm{h}, \mathrm{s}, \mathrm{s}, \mathrm{r}, \boldsymbol{\epsilon},(\mathrm{l}), \mathrm{m}, \mathrm{f}$ \\
\hline \multirow[t]{2}{*}{ Polish } & $\Xi_{\ell}$ & 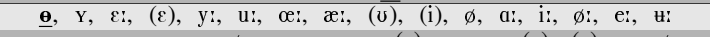 & $\underline{\mathbf{d}}, \mathrm{b}, \mathrm{j}, \mathrm{t}, \mathrm{s}, \mathrm{g}, \mathrm{v}, \mathrm{f}, \mathrm{h}, \mathrm{k},(\mathrm{f}),(\mathrm{j}), \mathrm{t}, \mathrm{s},(\mathrm{m})$ \\
\hline & $\Xi_{h_{\ell}}$ & 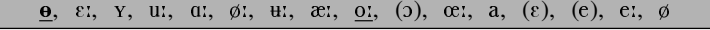 & $\underline{\mathbf{d}}, \mathrm{b}, \mathrm{t}, \mathrm{p}, \mathrm{g},(\mathrm{j}), \mathrm{k}, \mathrm{h}, \mathrm{s}, \mathrm{\epsilon}, \mathrm{s},(\mathrm{r}), \mathrm{t},(\mathrm{l}), \mathrm{v}$ \\
\hline \multirow[t]{2}{*}{ Russian } & $\Xi_{\ell}$ & 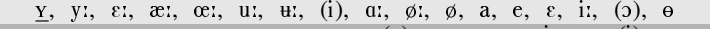 & $\underline{\mathbf{b}}, \mathbf{\mathbf { d }}, \mathrm{f}, \mathrm{t}, \mathrm{g},(\mathrm{m}), \mathrm{k}, \mathrm{s}, \mathrm{t},(\mathrm{r}), \mathrm{p},(\mathrm{s}), \mathrm{j}, \mathrm{v}, \quad(\mathrm{l})$ \\
\hline & $\Xi_{h_{\ell}}$ & 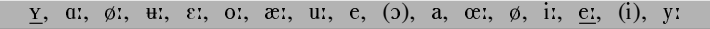 & $\underline{\mathbf{d}}, \underline{\mathbf{b}}, \mathrm{t}, \mathrm{p}, \mathrm{g}, \mathrm{k}, \mathrm{j},(\mathrm{s}),(\mathrm{r}), \mathrm{c}, \mathrm{h},(\mathrm{l}), \mathrm{s}, \mathrm{t},(\mathrm{m})$ \\
\hline \multirow[t]{2}{*}{ Greek } & $\Xi_{\ell}$ & 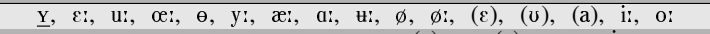 & $\underline{\mathbf{b}}, \underline{\mathbf{d}}, \mathrm{p}, \mathrm{t}, \mathrm{s}, \mathrm{f}, \mathrm{k}, \mathrm{t}, \mathrm{g},(\mathrm{r}), \mathrm{l},(\mathrm{j}), \mathrm{s}, \mathrm{j}, \mathrm{h}, \mathrm{c}$ \\
\hline & $\Xi_{h_{\ell}}$ & 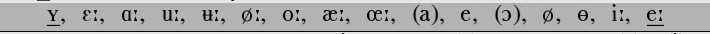 & $\underline{\overline{\mathbf{d}}}, \overline{\mathrm{p}}, \underline{\mathbf{b}}, \mathrm{t}, \mathrm{g}, \mathrm{k},(\mathrm{j}), \mathrm{t}, \mathrm{s}, \mathrm{h}, \mathrm{s},(\mathrm{r}), \mathrm{c}, \mathrm{l}, \mathrm{m}, \mathrm{f}$ \\
\hline \multirow[t]{2}{*}{ Spanish } & $\Xi_{\ell}$ & 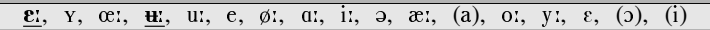 & $\underline{\mathbf{d}}, \mathrm{b}, \mathrm{j}, \mathrm{f}, \mathrm{t}, \mathrm{c}, \mathrm{k}$, (f), $\mathrm{g}, \mathrm{t}, \mathrm{p}, \mathrm{s}, \mathrm{s},(\mathrm{l}), \mathrm{j},(\mathrm{r}), \mathrm{h}$ \\
\hline & $\Xi_{h_{\ell}}$ & 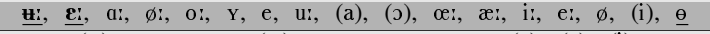 & $\underline{\underline{\mathbf{d}}}, \mathrm{b}, \mathrm{t}, \mathrm{p}, \mathrm{k}, \mathrm{g}, \mathrm{j}, \mathrm{\epsilon}, \mathrm{s}, \mathrm{h},(\mathrm{r}),(\mathrm{l}), \mathrm{s}, \mathrm{t}, \mathrm{m},(\mathrm{f}), \mathrm{f}$ \\
\hline \multirow[t]{2}{*}{ Turkish } & $\Xi_{\ell}$ & 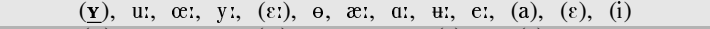 & $\underline{\mathbf{b}}, \underline{\mathbf{d}}, \mathrm{t},(\mathrm{s}), \mathrm{p}, \mathrm{j}, \mathrm{k}, \mathrm{t}, \mathrm{g}, \mathrm{r}, \mathrm{l}, \mathrm{s}, \mathrm{n}, \mathrm{j}, \mathrm{h}, \mathrm{c},(\mathrm{m}), \mathrm{f}$ \\
\hline & $\Xi_{h_{\ell}}$ & 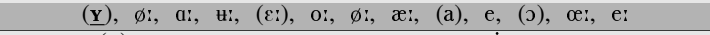 & $\underline{\underline{\mathbf{d}}}, \underline{\overline{\mathbf{b}}}, \mathrm{t}, \mathrm{p}, \mathrm{g}, \mathrm{k}, \mathrm{j}, \mathrm{t}, \mathrm{h}, \mathrm{s}, \mathrm{r},(\mathrm{s}), \mathrm{c}, \mathrm{l},(\mathrm{m}), \mathrm{f}, \mathrm{v}, \mathrm{j}$ \\
\hline \multirow[t]{2}{*}{ Arabic } & $\Xi_{\ell}$ & 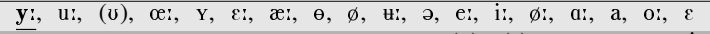 & $\underline{\mathbf{d}}, \mathrm{s}, \mathrm{f}, \mathrm{t}, \mathrm{j},(\underline{\mathbf{b}}),(\mathrm{g}),(\mathrm{h}), \mathrm{v}, \mathrm{c}, \mathrm{k}, \mathrm{r}, \mathrm{t}$ \\
\hline & $\Xi_{h_{\ell}}$ & y:, u:, y, œ:, æ: 甘:, ع:, а:, & $(\underline{\mathbf{b}}), \mathrm{t}, \mathrm{p},(\mathrm{g}), \mathrm{k}, \underline{\mathbf{d}}, \mathrm{s},(\mathrm{j}),(\mathrm{h}), \mathrm{\epsilon}, \mathrm{t}, \mathrm{r}, \mathrm{s}$ \\
\hline \multirow[t]{2}{*}{ Chinese } & $\Xi_{\ell}$ & 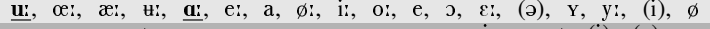 & $\underline{\mathbf{b}}, \mathrm{p}, \mathbf{\mathbf { d }}, \mathrm{t}, \mathrm{g}, \mathrm{k}, \mathrm{r}, \mathrm{t},(\mathrm{s}), \mathrm{s}, \mathrm{l}, \mathrm{j}, \mathrm{f}, \mathrm{(h)}, \mathrm{m}, \mathrm{n}, \mathrm{c}, \mathrm{f}$ \\
\hline & $\Xi_{h_{\ell}}$ & 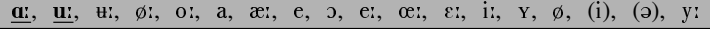 & $\underline{\mathbf{d}}, \mathrm{p}, \underline{\mathbf{b}}, \mathrm{t}, \mathrm{g}, \mathrm{k}, \mathrm{j}, \mathrm{(s)}, \mathrm{(h)}, \mathrm{r}, \mathrm{t}, \boldsymbol{\epsilon}, \mathrm{l}, \mathrm{s}, \mathrm{m}, \mathrm{f}, \mathrm{f}, \mathrm{v}$ \\
\hline \multirow[t]{2}{*}{ Persian } & $\Xi_{\ell}$ & 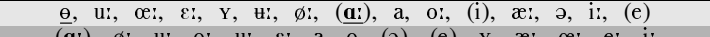 & $\underline{\mathbf{d}}, \mathrm{p}, \mathrm{b}, \mathrm{t}, \mathrm{g}, \mathrm{k}, \mathrm{t}, \mathrm{(h),}(\mathrm{j}), \mathrm{c}, \mathrm{s}, \mathrm{l}, \mathrm{s}, \mathrm{r}$, (f), (f) \\
\hline & $\Xi_{h_{\ell}}$ & & $\mathrm{k},(\mathrm{j}), \mathrm{g},(\mathrm{h}), \mathrm{s}, \mathrm{c}, \mathrm{t}, \mathrm{r}, \mathrm{l}, \mathrm{s}, \mathrm{m}, \mathrm{v}$ \\
\hline
\end{tabular}

ms. The central $i$ th subframe was used to compute the 12 static MFCCs and the other two subframes to compute the dynamic (12 $\Delta$ 's and $12 \Delta \Delta$ 's) MFCCs. To compute the MFCCs, the speech signal was first pre-emphasized and the output was windowed by a Hamming window. The DFT dimensionality was 512 and we considered 31 mel filters to compute the MFCC. The distorted speech vectors for each considered frame were generated by adding $30 \mathrm{~dB}$ SNR i.i.d. Gaussian noise in each time-domain speech signal frame, and a set of 100 perturbation vectors was computed. The speech corpus (a detailed description can be found in [1]), sampled at $16 \mathrm{kHz}$, was collected from 23 male and 14 female L2 learners of Swedish. The native part of the collection includes recordings from 9 male and 2 female Swedish speakers.

\section{Results}

We present the experimental outcome of our methods and compare them with firstly a linguistic study of problematic Swedish phonemes for different L1 groups [10] and secondly a listening test on the same data set, using 8 native speakers. It should be noted that the task for the automatic analysis and the listeners was exclusively oriented on judging if a phoneme was correctly or incorrectly pronounced without considering any potential grammatical, syntactical or contextual errors.

Table 1 lists the phonemes found to be difficult for the different groups of non-native speakers. $\Xi_{\ell}$ refers to the method without the LDM input, which we call the spectro-temporal nPAD $^{1}$, while $\Xi_{h_{\ell}}$ is the hybrid (spectro-temporal/LDM) nPAD. For each L2 speaker group, it is shown, in order, the most deviating vowels (to the left) and consonants (to the right) according to these methods. Divergences from the theoretical findings are reported in parentheses. For the vowels, the hybrid method improves the performance of the system for most of the language groups both in terms of quality, i.e., total number of seriously problematic phonemes found, and quantity, i.e., amount of mismatches with [10]. For example, the hybrid method captures all the seriously problematic vowels for English, French and Arabic speakers in comparison to only one group (English speakers) for the spectro-temporal method. In

\footnotetext{
${ }^{1}$ Comparing with the approach in [1], $\Xi_{\ell}$ is computed in spectrotemporal auditory and acoustic domains, instead of spectral only.
}

total, we get, for the spectro-temporal vs. the hybrid method, 30 vs. 26 mismatches out of totally 177 mispronunciations inventoried in [10], and 18 vs. 14 missed seriously mispronounced phonemes out of 127 . We also note that both methods accomplished better results in comparison to our previous approach, in which only the spectral cues of the speech signal were examined [1] (that method resulted in 34 mismatches and 27 missed seriously problematic vowels). For consonants, the addition of the LDM module did not influence the system's overall performance to the same extend as for vowels. The number of divergences from [10] was 33 for the spectro-temporal and 32 for the hybrid method, compared to 35 (out of 173 mispronunciations) for the spectral-only. The amount of seriously mispronounced consonants that were missed was 18 for the spectro-temporal and 24 for the hybrid method, compared to 22 out of 118 in [1].

Until now we have focused on revealing a pronunciation error without analyzing it. Table 2 shows the phonemes found misclassified by the LDM when using MMFCCs and MFCCs as observation vectors. The target phonemes are shown, in each case, at the top black row (vowels at the upper and consonants at the bottom part of the table) and the estimated misclassifications are shown below them, for every language group. Generally speaking, most of the results are in good agreement with Bannert's study, e.g., it is found that the Swedish retroflex /š is often erroneously pronounced as the alveolar /s/ by most nonnative groups. When comparing Tables 1 and 2 we see that all the phonemes that are detected by the LDM are listed in the problematic phonemes found by the perceptual-based methods.

For a more accurate evaluation, we also performed a listening test with 8 native listeners -3 'experts', i.e., teachers of Swedish as a foreign language and 5 ordinary, 'naive' listeners - on the same speech data as the automatic methods. The listeners, seated in front of a computer graphical interface program, were asked to evaluate (accept or reject) if a specific target phoneme was pronounced natively within various contexts (words or sentences). The examined phonemes were those vowels and consonants that the two nPAD evaluations, $\Xi_{\ell}$ and $\Xi_{h_{\ell}}$ found to be the most problematic. In Table 1, these phonemes are shown underlined. They are additionally in bold, when the judgment of the expert listening group agreed with the nPAD methods. We can see that for most of the vowels and almost 
Table 2: Phonemes that are found by the LDM classifier to have replaced the target ones are shown for each L2 group. A black bullet denotes no misclassification. In some cases, the MMFCCs and MFCCs give different results that are shown with slash separators.

\begin{tabular}{|c|c|c|c|c|c|c|c|c|c|c|c|c|c|c|c|c|c|c|}
\hline L1 bkgr. & \multicolumn{18}{|c|}{ vowels } \\
\hline German & e: & $\mathrm{a}$ & $\bullet / \mathrm{e}$ : & o: & כ/o: & $a / 2$ & $\mathrm{HI}$ & $\mathrm{H}$ & $\emptyset: \bullet$ & e: & $\mathrm{a}$ & $\bullet$ & a & $\bullet$ & $\bullet$ & $\bullet$ & $\bullet$ & $\bullet$ \\
\hline French & $a$ & $a / a$ & a/e: & o: & 2 & 2 & e:/it & $\mathrm{i}$ & a: & $\mathrm{a}$ & $\bullet$ & $\bullet$ & $\bullet$ & $a / \bullet$ & $\bullet / \mathrm{ar}$ & $\bullet$ & $\bullet$ & $\bullet$ \\
\hline Russian & 2 & $\mathrm{a}$ & a/e & $\mathrm{a}$ & o & $\mathrm{a} / \mathrm{r}$ & i: & $i / 2$ & a:/e: & $\mathrm{a}$ & $\bullet / a$ & $\bullet / a$ & $\bullet$ & $\mathrm{a}$ & $\bullet / \mathrm{e}:$ & $\bullet$ & $\bullet$ & $\bullet$ \\
\hline Greek & $a / 2$ & $a / 2$ & e: & $0: / \mathrm{a}:$ & 5 & 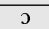 & it & $\mathrm{i}$ & $\varnothing: / \mathrm{a:}$ & $\phi:$ & $\bullet$ & $\bullet$ & $\bullet$ & $\bullet$ & $\bullet$ & $\bullet$ & $\bullet$ & $\bullet$ \\
\hline Spanish & 2 & $\mathrm{a}$ & e: & $\mathrm{a} / \mathrm{a}:$ & $\mathrm{a}$ & $\mathrm{a}$ & i: & $\mathrm{i}$ & a: & $\mathrm{a} / \mathrm{e}:$ & $\bullet$ & $\bullet$ & $\bullet$ & $\bullet$ & $\bullet$ & $\bullet$ & $\bullet$ & $\bullet$ \\
\hline Chinese & e: & $\mathrm{a} / \mathrm{el}$ & $\mathrm{a} / \mathrm{el}$ & $0: / a$ & 5 & $\mathrm{a}$ & u:/e: & e: $/ 1$ & $\varnothing: / \mathrm{a}:$ & $\mathrm{et}$ & $\bullet / a$ & $\bullet$ & $\bullet$ & $\bullet$ & $\bullet / \mathrm{e}$ & $\bullet$ & $\bullet$ & $\bullet$ \\
\hline Persian & כ/e: & $\mathrm{a}$ & $\bullet / \mathrm{e}:$ & $0: / 3$ & 0 & $\mathrm{a}$ & i: & $\mathrm{i}$ & a: & $\varnothing: / \mathrm{a}:$ & $\bullet / \mathrm{a}$ & $\bullet / 2$ & $\bullet$ & $\bullet$ & $\bullet$ & $\alpha:$ & $\bullet$ & $\bullet$ \\
\hline \multirow[t]{2}{*}{ L1 bkgr. } & \multicolumn{18}{|c|}{ consonants } \\
\hline & $\mathrm{n}$ & d & b & $\mathrm{h}$ & g & $\mathrm{n}$ & $\mathrm{v}$ & $\mathrm{j}$ & t & fo & $\mathrm{p}$ & s. & $\mathrm{k}$ & 6 & $\mathrm{~m}$ & $\mathrm{~s}$ & & \\
\hline Eng.(US) & $\mathrm{m} / \bullet$ & $t$ & $t$ & $\mathrm{r} / \mathrm{k}$ & $\mathrm{k}$ & $\mathrm{m} / \mathrm{n}$ & $\mathrm{r}$ & $\mathrm{J} / \mathrm{r}$ & $t / k$ & $f / k$ & $\bullet / t$ & $\bullet / 6$ & $\bullet$ & $\bullet$ & $\bullet$ & $\bullet$ & & \\
\hline German & $\mathrm{m} / \bullet$ & $\mathrm{t}$ & $\mathrm{t}$ & $f / k$ & $\mathrm{k}$ & $\mathrm{m} / \mathrm{a}$ & $\mathrm{k} / \mathrm{r}$ & $\mathrm{i} / \bullet$ & $t$ & $\mathrm{~s} / \mathrm{f}$ & $t$ & $s / 6$ & $\bullet$ & $\bullet$ & $\bullet$ & $\bullet$ & & \\
\hline Turkish & $\mathrm{m} / \bullet$ & $t$ & $\mathrm{~m}$ & $\mathrm{k}$ & $\mathrm{k} / \mathrm{r}$ & $\mathrm{i}: \mathrm{n}$ & $5 / \bullet$ & $\bullet$ & $t$ & $f / s$ & $t$ & $\mathrm{~s}$ & $\bullet$ & $\mathrm{s} / \bullet$ & $\bullet$ & $\bullet$ & & \\
\hline Arabic & $\mathrm{m} / \mathrm{a}$ & $\mathrm{J} / \mathrm{t}$ & $\mathrm{v} / \mathrm{p}$ & $1 / \mathrm{t}$ & $\mathrm{k} / \mathrm{l}$ & $0:$ & $\bullet / \mathrm{r}$ & $\mathrm{J} / \mathrm{r}$ & $t$ & $\mathrm{r} / \mathrm{s}$ & $t$ & $\mathrm{~s}$ & $\bullet$ & $\mathrm{s}$ & $\bullet / \mathrm{n}$ & $f / \bullet$ & & \\
\hline Chinese & $\bullet$ & $t$ & $\mathrm{t}$ & $\mathrm{k} / \mathrm{t}$ & $\mathrm{k}$ & $i: / n$ & $\bullet / t$ & $\bar{i}$ & $t / k$ & $\mathrm{f} / \mathrm{s}$ & $\bar{t}$ & $\mathrm{~s}$ & $\bullet$ & $\mathrm{s} / \bullet$ & $\bullet / \mathrm{n}$ & $\bullet$ & & \\
\hline Persian & $\bullet$ & $t$ & $\mathrm{t}$ & $t$ & $\mathrm{k}$ & $\mathrm{n}$ & $\bullet / t$ & $\mathrm{~J} / \mathrm{r}$ & $t$ & $f / k$ & $t$ & $s / 6$ & $\bullet$ & $\mathrm{s}$ & $\bullet$ & $\bullet$ & & \\
\hline
\end{tabular}

all of the consonants that were tested, the human experts and the automatic evaluations coincide. In addition, we considered some of the phonemes that were expected to have caused serious problems for specific L2 groups, according to the linguistic study [10], but for which the automatic methods found no mispronunciation. These phonemes were the Swedish $/ 1 /$ for the English L2 group, /n/ for the Russian, /n/ for the Greek, /v/ for Spanish, /i:/ for Turkish, /f/ for Arabic, / $\mathrm{y} /$ and $/ \Theta /$ for the Chinese and $/ \mathrm{n} /$ and $/ \mathrm{y}: /$ for the Persian group. In all cases, the human listeners agreed with the nPAD approaches that the target phonemes were produced in a natively manner with a small exception for the Persian /y:/, for which two experts indicated an error. Finally, Table 3 shows the performance of each of the three methods as compared to the human listeners' assessment for the examined phonemes. We see that the judgment of the automatic methods are closer to that of the Swedish teachers, which is a positive finding since we aim at integrating our methods in a computer-assisted pronunciation training program and hence want to achieve an evaluation corresponding to that of human teachers. In addition, it seems that the method that is purely based on the perceptual knowledge is closer to the experts' opinion. We note however that the listening test included a relatively small number of phonemes and listeners, since the goal was not to perform a hard evaluation of the proposed methods but rather to obtain a verification on their reliability.

\section{Conclusions}

We have modified and improved the performance of our previously presented perceptual based system thereby including dynamic information from both a spectro-temporal auditory model and a linear dynamic system. In addition, we examined the task as a classical classification problem not only to identify but also to stochastically analyze the errors. A listening test with native listeners on the same data has verified the effectiveness of our methods, which is important for our future plans to integrate the system into a pronunciation training program.

\section{Acknowledgments}

This work is supported by the Swedish Research Council project 80449001 CALATEA. We wish to thank all native lis-
Table 3: The percentage of the automatic method findings that are verified by native listener groups.

\begin{tabular}{c|c|c|c} 
native & \multicolumn{3}{|c|}{ automatic methods } \\
listener group & spectro-temporal $\Xi_{\ell}$ & hybrid $\Xi_{h_{\ell}}$ & LDM classifier \\
\hline expert & $86.5 \%$ & $69.2 \%$ & $63.5 \%$ \\
naive & $53.8 \%$ & $36.5 \%$ & $34.6 \%$ \\
\hline
\end{tabular}

teners that participated in this study.

\section{References}

[1] C. Koniaris and O. Engwall, "Phoneme Level Non-Native Pronunciation Analysis by an Auditory Model-based Native Assessment Scheme", in Interspeech, Florence, Italy, 1157-1160, Aug. 2011.

[2] K. Sjölander, "An HMM-based System for Automatic Segmentation and Alignment of Speech", in Fonetik, 93-96, Jun. 2003.

[3] V. Digalakis, J. R. Rohlicek, and M. Ostendorf, "ML Estimation of a Stochastic Linear System with the EM Algorithm and Its Application to Speech Recognition", IEEE Trans. Speech, Audio Proc., 1(4):431-442, Oct. 1993.

[4] S. Chatterjee, C. Koniaris and W. B. Kleijn, "Auditory Model based Optimization of MFCCs Improves Automatic Speech Recognition Performance", in Interspeech, Brighton, UK, 2987 2990, Sep. 2009.

[5] S. B. Davis and P. Mermelstein, "Comparison of Parametric Representations for Monosyllabic Word Recognition in Continuously Spoken Sentences", IEEE Trans. Acoust., Speech, Sig. Proc., 28(4):357-366, Aug. 1980

[6] S. van de Par, A. Kohlrausch, G. Charestan and R. Heusdens, "A New Psychoacoustical Masking Model for Audio Coding Applications", in IEEE Int. Conf. Acoust., Speech, Sig. Proc., Orlando, FL, USA, 2:1805-1808, May 2002.

[7] H. E. Rauch, F. Tung and C. T. Striebel, "Maximum Likelihood Estimates of Linear Dynamic Systems", AIAA Journal, 3(8):14451450, Aug. 1965.

[8] R. E. Kalman, "A New Approach to Linear Filtering and Prediction Problems", Tr. ASME, J. Basic Eng., 82(D):35-45, Mar. 1960

[9] T. Dau and D. Püschel and A. Kohlrausch, "A quantitative model of the effective signal processing in the auditory system. I. Model structure", J. Acoust. Soc. Amer., 99(6):3615-3622, Jun. 1996.

[10] R. Bannert, "Problems in Learning Swedish Pronunciation and in Understanding Foreign Accent", Folia Linguistica, 18(1-2):193222, Jan. 1984. 